\title{
Melatonin reduces oxidative damage in mouse granulosa cells via restraining JNK-dependent autophagy
}

\author{
Yan Cao*, Ming Shen*, Yi Jiang, Shao-chen Sun and Honglin Liu \\ College of Animal Science and Technology, Nanjing Agricultural University, Nanjing, China \\ Correspondence should be addressed to M Shen or H Liu; Email: shenm2015@njau.edu.cn or liuhonglin@njau.edu.cn
}

*(Y Cao and M Shen contributed equally to this work)

\begin{abstract}
Oxidative stress-induced granulosa cell (GCs) injury is believed to be a common trigger for follicular atresia. Emerging evidence indicates that excessive autophagy occurs in mammalian cells with oxidative damage. $\mathrm{N}$-acetyl-5-methoxytrypamine (melatonin) has been shown to prevent GCs from oxidative injury, although the exact mechanism remains to be elucidated. Here, we first demonstrated that the suppression of autophagy through the JNK/BCL-2/BECN1 signaling is engaged in melatonin-mediated GCs protection against oxidative damage. Melatonin inhibited the loss of GCs viability, formation of GFP-MAP1LC3B puncta, accumulation of MAP1LC3B-II blots, degradation of SQSTM1 and the expression of BECN1, which was correlated with impaired activation of JNK during oxidative stress. On the other hand, blocking of autophagy and/or JNK also reduced the level of $\mathrm{H}_{2} \mathrm{O}_{2}-$ induced GCs death, but failed to further restore GCs viability in the presence of melatonin. Particularly, the suppression of autophagy provided no additional protective effects when GCs were pretreated with JNK inhibitor and/or melatonin. Importantly, we found that the enhanced interaction between BCL-2 and BECN1 might be a responsive mechanism for autophagy suppression via the melatonin/ JNK pathway. Moreover, blocking the downstream antioxidant system of melatonin using specific inhibitors further confirmed a direct role of melatonin/JNK/autophagy axis in preserving GCs survival without scavenging reactive oxygen species (ROS). Taken together, our findings uncover a novel function of melatonin in preventing GCs from oxidative damage by targeting JNK-mediated autophagy, which might contribute to develop therapeutic strategies for patients with ovulation failure-related disorders.

Reproduction (2018) 155 307-319
\end{abstract}

\section{Introduction}

Macroautophagy (referred to here as autophagy) is an evolutionary conserved catabolic pathway in eukaryotes that involves recycling of cellular materials, including long-lived proteins and organelles (Levine \& Klionsky 2004). Autophagy is initiated by the formation of double-membrane vesicles called autophagosomes that sequester and deliver cytoplasmic components to the lysosome for degradation. Autophagy occurs at a basal rate in normal cells, removing damaged cellular content to maintain homeostasis (Levine \& Kroemer 2008). During periods of starvation, stimulation of autophagy is an autonomous cell survival mechanism, which provides nutrients to maintain metabolism and ATP levels compatible with cell survival (Mehrpour et al. 2010). In the context of cancer, activation of autophagy by hypoxia or drug treatment has also been shown to promote tumor cell survival (Sui et al. 2013). However, there is considerable evidence suggesting the role of autophagy in repressing cell viability (Tsujimoto \& Shimizu 2005). Almost paradoxically, autophagy has been described as a type of apoptosis-independent cell death (also known as type II programmed cell death), which is characterized by autophagosome accumulation in the absence of apoptotic bodies and cleaved CASP3 (Kroemer \& Levine 2008, Gustafsson \& Gottlieb 2009, Nishida et al. 2009).

In mammalian ovaries, only a few follicles are selected for ovulation, while most of them ( $\geq 99 \%)$ undergo a degenerative process termed follicular atresia (Kaipia \& Hsueh 1997). Reactive oxygen species (ROS) are free radicals derived from cellular metabolism or environmental stimuli. Unlimited ROS generation shift cells into a state of oxidative stress, leading to destructive effects on cellular components (Birben et al. 2012). Previous studies indicated that increased oxidative damage in granulosa cells (GCs) is closely correlated to the occurrence of follicular atresia (Murdoch 1998), which may give rise to certain anovulatory disorders, such as polycystic ovary syndrome and premature ovarian failure (Agarwal et al. 2012). In contrast, impaired ROS production has been shown to alleviate GC injury in atretic follicles (Tilly \& Tilly 1995, Gupta et al. 2006). Notably, recent evidence suggested that autophagy may be responsible for oxidative stress-induced GC damage during follicular atresia (Duerrschmidt et al. 2006, 
Serke et al. 2009). In fact, our previous work has provided more direct evidence demonstrating the role of oxidative stress in promoting autophagic GC death (Shen et al. 2017). Therefore, discovery and identification of an antioxidant agent by targeting autophagy may provide benefits to clinical therapy for anovulatory disorders caused by inappropriate follicular atresia.

$\mathrm{N}$-acetyl-5-methoxytryptamine (melatonin), the main secretory product of the pineal gland, plays diverse functions in regulating circadian rhythms, inflammation, carcinogenesis, immune response and ROS clearance (Manchester et al. 2015, Reiter et al. 2016). Growing evidence suggested that melatonin also serves as a prosurvival factor for antral follicles (Tamura et al. 2009). It stimulates GCs to synthesize sex steroid hormones and promotes the progression of follicular maturation (Tamura et al. 2009). In contrast, inhibiting melatonin production by pinealectomy significantly increased the number of atretic follicles in mammalian ovaries (Soares et al. 2003b). Interestingly, it was claimed that melatonin in the follicular fluid may be directly produced by GCs, whereas melatonin in turn enhances the antioxidant capacity of GCs by upregulating gene expression of SOD, GPx and CAT (Tamura et al. 2009). As previously mentioned, excessive ROS induces GC death and the resultant follicular atresia. Increased levels of melatonin in follicular fluid would normally protect GCs from free radical damage and suppress atresia through its direct and indirect antioxidant actions (Tamura et al. 2013). However, there are few further reports investigating the downstream signaling of melatonin for GCs protection during oxidative stress.

BECN1, the mammalian homolog of yeast ATG6, is a platform protein that recruits several different partners to control the activity of PIK3C3 (class III phosphatidylinositol 3-kinase), which catalyzes the conversion of phosphatidylinositol into phosphatidylinositol 3-phosphate, a lipid required for autophagosome formation in response to ATG proteins (Levine \& Yuan 2005). As a BH3-only protein, BECN1 also interacts with other BCL-2 family members, such as BCL-2, BCL-XL, BCL-W and MCL-1 (Erlich et al. 2007). Previous studies indicated that dissociation of $B C L-2$ from BECN1 may be an important mechanism for autophagy induction under stress conditions (Pattingre et al. 2005). JNK (also known as c-Jun N-terminal kinase), a member of the MAPKs (mitogen-activated protein kinases), regulates diverse biological processes including proliferation, differentiation, senescence, autophagy, metabolism and stress response (Weston \& Davis 2007). Further investigations suggested that the activation of JNK by nutrient deprivation could inhibit the interactions between BCL-2 and BECN1 (Wei et al. 2008). Moreover, melatonin has been shown to reduce hepatic ischemia/ reperfusion injury by suppressing the JNK pathway (Liang et al. 2009). These findings thus implicate a possible relationship among melatonin, JNK and the autophagic machinery. Nevertheless, it remains unclear whether autophagy is correlated with JNK signaling in oxidative stress-induced GCs death and the protective role of melatonin in this process.

Herein, we investigated whether the preventive effects of melatonin on oxidative stress-induced GC injury is associated with autophagy signaling. We specifically focused on the BCL-2/BECN1 complex and upstream pathway activity, including JNK activation. Furthermore, we determined whether melatonin could attenuate oxidative stress-induced GCs toxicity via this signaling cascade.

\section{Materials and methods}

\section{Chemicals and antibodies}

PBS was purchased from Gibco. Pregnant mare serum gonadotropin (PMSG) was purchased from Ningbo Second Hormone Factory (Ningbo, Zhejiang, China). Melatonin, SP600125, 3-methyladenine (3-MA) and NSC 23766 were from Selleck Chemicals (Houston, TX, USA). $\mathrm{H}_{2} \mathrm{O}_{2}$, anti-MAP1LC3B and anti-TUBA1A were bought from Sigma-Aldrich. L-Buthionine sulfoximine, 3-amino-1,2,4triazole, diethyldithiocarbamic acid sodium salt trihydrate, mercaptosuccinic acid, Luzindole and Map2k7 siRNA were purchased from Santa Cruz Biotechnology. Active Rac1 Detection Kit and antibodies against BCL-2, BECN1, Phospho-MAP2K7 and MAP2K7 were obtained from Cell Signaling Technology. SQSTM1 antibody was purchased from Abcam.

\section{Animals}

All procedures with mice were conducted in accordance with the guidelines of the Animal Research Institute Committee at Nanjing Agricultural University. Three week-old female ICR mice (Qing Long Shan Co., Animal Breeding Center, Nanjing, China) were injected intraperitoneally with 10 IU PMSG. After $48 \mathrm{~h}$, all mice were killed by cervical dislocation, and their ovaries were harvested for in vitro experiments. Prior to killing, the mice were housed five per cage in a temperature-controlled $\left(22 \pm 2^{\circ} \mathrm{C}\right)$ room with 12:12-h light:darkness cycles (lights on from 07:00 h to 19:00 h); they had ad libitum access to water and food. All experimental protocols were approved by the Committee of Animal Research Institute, Nanjing Agricultural University, China.

\section{Cell collection and treatments}

Primary GCs were collected as described (Shen et al. 2012). Briefly, superovulated mouse ovaries were harvested and individually transferred into $35 \mathrm{~mm}$ Petri dishes containing PBS, and then punctured with a syringe to release GCs (a mixture of mural and cumulus cells) from pre-ovulatory follicles under a surgical dissecting microscope (Olympus). For drug administration, GCs pretreated with melatonin $(10 \mu \mathrm{M})$ for $24 \mathrm{~h}$ were washed in PBS and incubated 
with medium containing $0.2 \mathrm{mM} \mathrm{H}_{2} \mathrm{O}_{2}$ for $2 \mathrm{~h}$. In some experiments, GCs were treated with SP600125 $(25 \mu \mathrm{M})$ or 3-MA (10 mM) $1 \mathrm{~h}$ before $\mathrm{H}_{2} \mathrm{O}_{2}$ exposure. For the suppression of melatonin-mediated antioxidative effects, a cocktail consists of inhibitors (hereafter referred to as antioxidant inhibitors or AOI) against glutathione/GSH (L-Buthionine sulfoximine; $1.5 \mathrm{mM}$ ), catalase/CAT (3-Amino-1,2,4-triazole; $10 \mathrm{mM}$ ), superoxide dismutase/SOD (diethyldithiocarbamic acid sodium salt trihydrate, $5 \mathrm{mM}$ ), glutathione peroxidase/ GPx (mercaptosuccinic acid, $5 \mathrm{mM}$ ) and glutathione reductase/GR (mercaptosuccinic acid, $5 \mu \mathrm{M}$ ) were added $1 \mathrm{~h}$ prior to $\mathrm{H}_{2} \mathrm{O}_{2}$ incubation. For the inhibition of melatonin receptors, cells were incubated with $10 \mu \mathrm{M}$ Luzindole for $2 \mathrm{~h}$ before melatonin treatment.

\section{Cell transfection}

Transfection was performed using Lipofectamine 3000 reagent (Invitrogen) according to supplier's instructions. Briefly, lipoplexes were prepared by combining $1 \mu \mathrm{L}$ P3000 reagent with $0.5 \mu \mathrm{g}$ of plasmid DNA suspended in $25 \mu \mathrm{L}$ of serumfree media and incubated for $5 \mathrm{~min}$ at room temperature. This solution was then combined with $1.5 \mu \mathrm{L}$ of Lipofectamine 3000 in $25 \mu \mathrm{L}$ serum-free media and further incubated for $5 \mathrm{~min}$ at room temperature. The mixture was then diluted into serum-free media to produce the desired concentration in a total volume of $0.5 \mathrm{~mL}$ and incubated with the cells at $37^{\circ} \mathrm{C}$ with $5 \% \mathrm{CO}_{2}$ for $48 \mathrm{~h}$.

\section{Confocal imaging of autophagic vacuoles}

The GFP-tagged microtubule-associated protein 1 light chain 3 beta (MAP1LC3B) expression vector was kindly provided by Prof. Jiyong Zhou (Zhejiang University, Hangzhou, China). The GFP-MAP1LC3B expression plasmid was transfected into GCs grown on coverslips. After $24 \mathrm{~h}$, cells were cultured in medium with or without $10 \mu \mathrm{M}$ melatonin for another $24 \mathrm{~h}$, rinsed using PBS and then exposed to $2 \mathrm{~h}$ of $\mathrm{H}_{2} \mathrm{O}_{2}$ incubation. The distribution and fluorescence emitted by GFP-MAP1LC3B puncta were then observed under a laser-scanning confocal microscope (Carl Zeiss, Zeiss LSM 710 META). Experiments were repeated three times. The fields of each coverslip were divided into 9 component squares, and 3 of them were randomly selected for counting GFP-MAP1LC3B-stained puncta at a magnification of $400 \times$.

\section{Cell viability assay}

Cell viability was assessed by measuring the conversion of tetrazolium salt (WST-8) to formazan according to the manufacturer's instructions (Dojindo Laboratories, Kumamoto, Japan). Briefly, cells seeded in 96-well plate were exposed to $\mathrm{H}_{2} \mathrm{O}_{2}$ incubation following melatonin, SP600125, AOI or 3-MA administration as indicated. After treatment, $10 \mu \mathrm{L}$ of CCK-8 solution were added to each well containing $100 \mu \mathrm{L}$ medium and incubated for an additional $2 \mathrm{~h}$ at $37^{\circ} \mathrm{C}$. Cell viability was then determined by reading the optical density at $450 \mathrm{~nm}$ using a microplate reader (Thermo Fisher Scientific).

\section{Measurement of JNK activity}

The activity of total JNK and JNK1 was determined using a JNK Activity Assay Kit (Genmed, Shanghai, China) as previously described (Weng et al. 2016). Briefly, cytosolic extracts were centrifuged at $16,000 \mathrm{~g}$ at $4{ }^{\circ} \mathrm{C}$ for $10 \mathrm{~min}$, and the protein concentrations were determined using a Bradford Protein Assay Kit (Beyotime Institute of Biotechnology, Shanghai, China). $50 \mu \mathrm{g}$ of total protein was incubated with $10 \mu \mathrm{L}$ chromophoric substrate at $37^{\circ} \mathrm{C}$. The optical density of each sample was immediately detected at $340 \mathrm{~nm}$ using a microplate reader (Thermo Fisher Scientific) and recorded 2 times at 5-min intervals.

\section{RAC1 activation assay}

The levels of active RAC1 were measured using Active RAC1 Detection Kit (Catalog number 8815, Cell Signaling Technology) according to the manufacturer's instructions. Briefly, $500 \mu \mathrm{g}$ of GCs lysates were incubated with GST-PAK1PBD fusion protein beads to enrich active GTP-bound RAC1, which were then immunoprecipitated with glutathione resin. Additional lysates were used as control inputs to determine total RAC1 levels. The proteins on the beads or the total cell lysates were subjected to immunoblot analysis using kitprovided RAC1 primary antibody.

\section{Detection of ROS generation}

ROS levels were determined by measuring the oxidative conversion of dichlorodihydrofluorescein (DCFH) to dichlorofluorescein (DCF), which generates green fluorescence upon excitation at $488 \mathrm{~nm}$. The experiments were conducted using the Intracellular ROS green Fluorescence Determination Kit (Beyotime Institute of Biotechnology) according to the manufacturer's directions. Fluorescent images were obtained using a Carl Zeiss laser-scanning confocal microscope (Carl Zeiss, Zeiss LSM 710 META). The optical density was evaluated in each GC with Imagej $1.42 q$ software (National Institutes of Health).

\section{qRT-PCR assay}

Total RNA was isolated with TRIzol reagent (Invitrogen) and reverse transcribed into cDNA using the Reverse Transcription System (TaKaRa) according to the manufacturer's instructions. Real-time PCR was performed with SYBR Premix ExTaq (TaKaRa) and genespecific primers (Table 1 for primer sequences) on the ABI StepOne PCR system (Applied Biosystems). Melting curves were analyzed to verify amplification specificity. Expression data were normalized to the amount of Actb expressed.

\section{Western blotting}

Western blotting was performed as described previously (Shen et al. 2014). Briefly, total protein extracts were fractioned by electrophoresis on a $12 \%$ ExpressPlus PAGE gel (Genscript, Nanjing, China) and transferred to PVDF membranes (Millipore) by electroblotting. Nonspecific binding sites were 
Table 1 The forward and reverse primers used for qRT-PCR.

\begin{tabular}{lcll}
\hline & & \multicolumn{2}{c}{ Primer sequence $\left(5^{\prime} \rightarrow 3^{\prime}\right)$} \\
\cline { 3 - 4 } Gene name & GenBank accession no. & Forward & Reverse \\
\hline$B C l-2$ & NM_009741.3 & GTGGATGACTGAGTACCTGAACC & AGCCAGGAGAAATCAAACAGAG \\
Becn 1 & NM_019584.3 & AGTTGCCGTTATACTGTTCT & TCAATCTTGCCTTTCTCC \\
Nctb & NM_007393.5 & GCTGTCCCTGTATGCCTCT & GTCTTTACGGATGTCAACG \\
\hline
\end{tabular}

blocked with $5 \%$ bovine serum albumin in TBST (Solarbio, Beijing, China) for $1 \mathrm{~h}$. The membranes were then incubated with primary antibodies $(1: 1000)$ against MAP1LC3B, SQSTM1, TUBA1A, BCL-2 and BECN1 in blocking solution overnight followed by a secondary antibody conjugated with horseradish peroxidase $(1: 2000)$ for another $1 \mathrm{~h}$. Detection of the bands was performed using the SuperSignal West Pico chemiluminescent substrate (Pierce). The expression values for target proteins were normalized to TUBA1A as the control for loading.

\section{Immunoprecipitation}

After washing with PBS, GCs were lysed in $1 \mathrm{~mL}$ cold IP lysis buffer (Pierce) containing a complete protease inhibitor cocktail (Roche). Whole-cell lysates were used for immunoprecipitation with anti-BCL-2. We added 2-4 mg of antibody to $1 \mathrm{~mL}$ of cell lysate, which was incubated at $4{ }^{\circ} \mathrm{C}$ overnight. After the addition of Protein A/G Plus Agarose beads (Beyotime Institute of Biotechnology), the incubation was continued for $1 \mathrm{~h}$. Immunoprecipitates were extensively washed with lysis buffer, eluted with SDS loading buffer (SunShineBio, Nanjing, China) by boiling for $5 \mathrm{~min}$ and then processed for immunoblotting with the indicated antibodies. The amount of coimmunoprecipitated proteins for each IP reaction was normalized to TUBA1A content in the whole-cell lysates (input).

\section{Statistical analysis}

Statistical significance was determined using the SPSS, version 16.0 software (SPSS). All experiments were repeated at least three times. Data were presented as means \pm S.E. The statistical significance between groups was analyzed using one-way ANOVA, and $P$ values less than 0.05 were considered to be statistically significant.

\section{Results}

\section{Melatonin counteracted oxidative injury in mouse granulosa cells (GCs) via inhibiting the JNK signaling}

Using an established in vitro model for the induction of oxidative damage in GCs, we found that $\mathrm{H}_{2} \mathrm{O}_{2}$ significantly decreased cell survival in a dose-dependent manner (Fig. 1A). According to the results, $\mathrm{H}_{2} \mathrm{O}_{2}$ at $0.2 \mathrm{mM}$ tended to provide a maximum useful dose for quantifying protective vs harmful response, and thus was chosen for subsequent experiments. To test whether melatonin protects GCs from oxidative injury, the CCK-8 assay was performed to measure cell viability. As shown in Fig. 1B, GCs pretreated with $10 \mu \mathrm{M}$ melatonin for $24 \mathrm{~h}$ exhibited an apparent resistance to $\mathrm{H}_{2} \mathrm{O}_{2}$-induced cell death. Notably, melatonin did not significantly alter the viability of cells under normal growth conditions (Fig. 1B), indicating that melatonin itself had no side effects on GC survival.
A

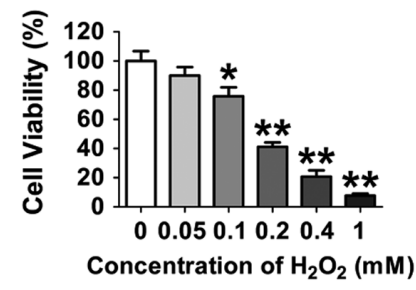

C

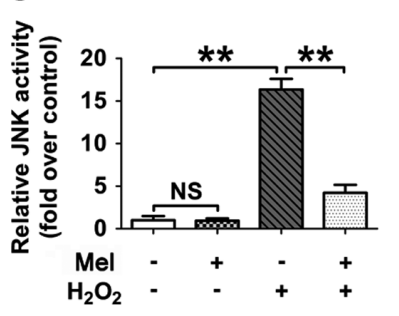

E

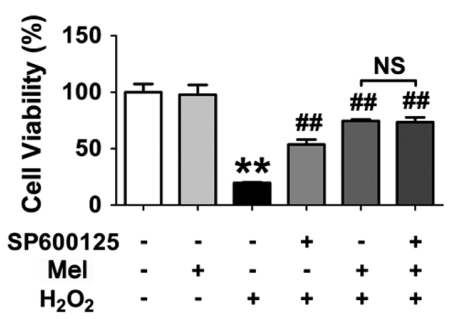

B

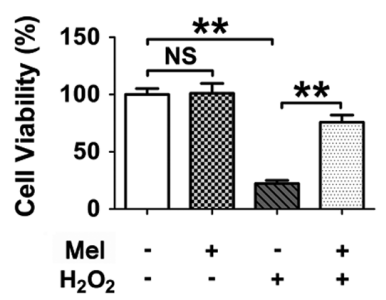

D

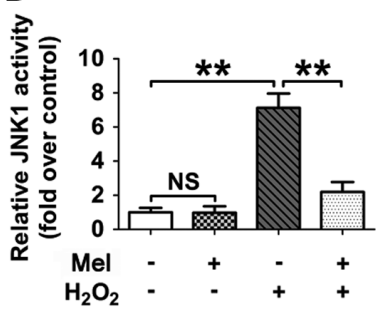

Figure $1 \mathrm{JNK}$ is involved in melatonin-mediated GC protection during oxidative stress. (A) Primary cultured GCs were incubated with $\mathrm{H}_{2} \mathrm{O}_{2}$ in various concentrations (from $0.05 \mathrm{mM}$ to $1 \mathrm{mM}$ ) for $2 \mathrm{~h}$. Cell viability was then determined using CCK-8 assay. Data represent mean \pm S.E.; $n=3$ in each group. *Represents $P<0.05$ vs control group. **Represents $P<0.01$ vs control group. (B) GCs were pretreated with or without melatonin $(10 \mu \mathrm{M})$ for $24 \mathrm{~h}$, rinsed in PBS, exposed to $2 \mathrm{~h}$ of $\mathrm{H}_{2} \mathrm{O}_{2}(0.2 \mathrm{mM})$ incubation, and then processed for the detection of cell viability (B), JNK activity (C) or JNK1 activity (D). Data represent mean \pm S.E.; $n=3$. ${ }^{* *} P<0.01$; NS, not significant, $P>0.05$. (E) GCs cultured in medium containing $10 \mu \mathrm{M}$ melatonin for $24 \mathrm{~h}$ were washed in PBS, and then subjected to $2 \mathrm{~h}$ of $\mathrm{H}_{2} \mathrm{O}_{2}(0.2 \mathrm{mM})$ exposure. For the inhibition of JNK activity, SP600125 (25 $\mu \mathrm{M})$ was added $1 \mathrm{~h}$ prior to $\mathrm{H}_{2} \mathrm{O}_{2}$ treatment. Data represent mean \pm S.E.; $n=3$. **Represents $P<0.01$ vs control group. ${ }^{\#}$ Represents $P<0.01$ vs $_{2} \mathrm{O}_{2}$ group. NS, not significant, $P>0.05$. 
c-Jun N-terminal kinase (JNK), a major effector of cellular stress response signaling, has been reported to be activated upon oxidative stimulation (Weston \& Davis 2007). To evaluate whether the protective effect of melatonin on $\mathrm{H}_{2} \mathrm{O}_{2}$-triggered $\mathrm{GC}$ injury is correlated with the inhibition of JNK, its activity was determined by measuring the oxidative conversion of NADH to NAD, which was detectable at $340 \mathrm{~nm}$ under a microplate reader. Significantly, oxidative stress-induced activation of total JNK and JNK1 isoform was abolished in the presence of melatonin (Fig. 1C and D).

RAC1, a small GTPase, plays essential roles in the cellular response to multiple types of stresses (Ozaki et al. 2000). Here, we examined whether RAC1 might be involved in oxidative stress-induced JNK activation in GCs. As expected, cells with $\mathrm{H}_{2} \mathrm{O}_{2}$ incubation exhibited a significant increase in JNK activity (Fig. 2C). However, GCs pretreated with melatonin or RAC1 inhibitor NSC 23766 blocked $\mathrm{H}_{2} \mathrm{O}_{2}$-induced JNK activation (Fig. 2C). Correspondingly, both melatonin and NSC 23766 repressed the upregulation of JNK activity upon oxidative stimulation, but RAC1 showed no additional inhibitory effect on JNK activation in cell received melatonin treatment (Fig. $2 \mathrm{C}$ ), indicating that the suppression of RAC1 might contribute to melatoninmediated JNK inhibition. MAP2K7 has been reported to stimulate JNK activation in response to extracellular stimuli (Moriguchi et al. 1997). To explore any influence of MAP2K7 on JNK activity in response to $\mathrm{H}_{2} \mathrm{O}_{2}$ or melatonin treatment, we blocked MAP2 $\mathrm{K} 7$ expression in cultured GCs using RNA interference (Fig. 2D). The $\mathrm{H}_{2} \mathrm{O}_{2}$ exposure facilitated MAP2K7 activation as suggested by increased phosphorylation, which was impaired in cells subjected to melatonin treatment or Map2k7 siRNA transfection (Fig. 2D and Supplementary Fig. 1C, see section on supplementary data given at the end of this article). Consistently, both MAP2K7 knockdown or melatonin incubation significantly repressed $\mathrm{H}_{2} \mathrm{O}_{2}$ stimulated JNK activation (Fig. 2F). However, Map2k7 siRNA did not further inhibit JNK activity in GCs pretreated with melatonin (Fig. 2F). Therefore, these data suggested a possible regulation by $\mathrm{H}_{2} \mathrm{O}_{2}$ and melatonin
A

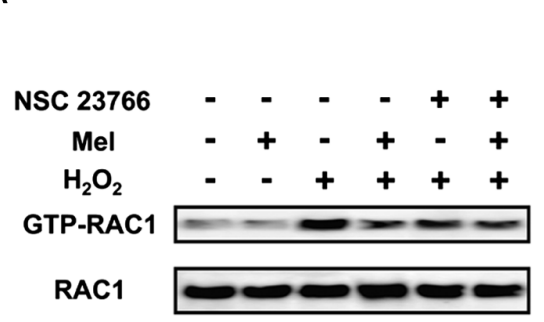

D

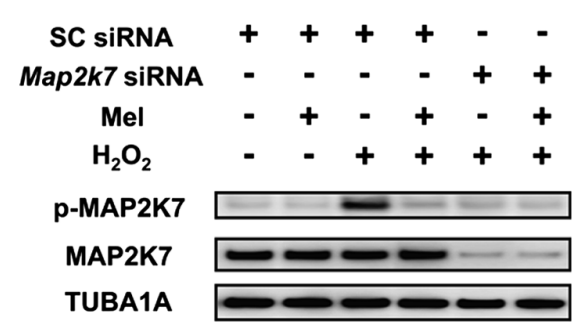

B

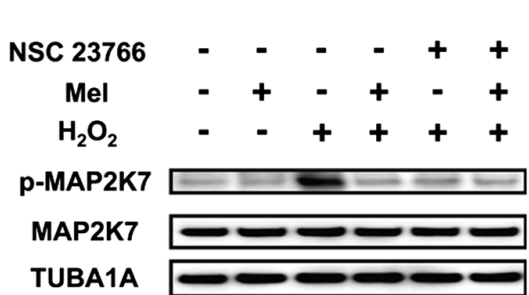

E

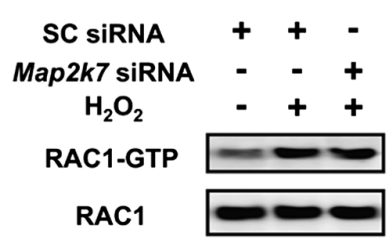

C

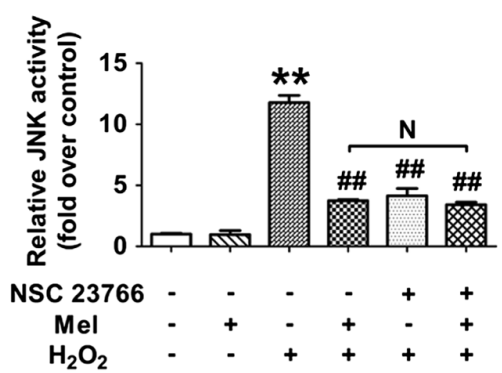

$\mathrm{F}$

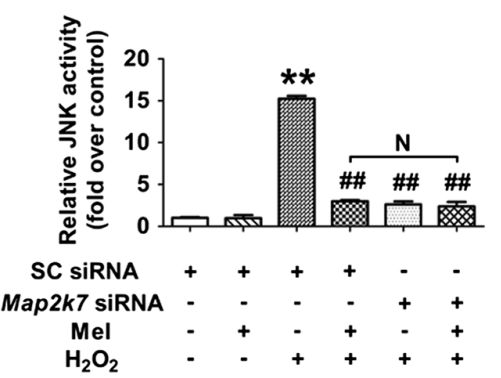

Figure $2 \mathrm{H}_{2} \mathrm{O}_{2}$ activates JNK through the RAC1/MAP2K7 pathway, which is inhibited by melatonin treatment. (A) GCs grown in medium containing $10 \mu \mathrm{M}$ melatonin for $24 \mathrm{~h}$ were rinsed using PBS, and then incubated with $0.2 \mathrm{mM} \mathrm{H}_{2} \mathrm{O}_{2}$ for $2 \mathrm{~h}$. For the inhibition of RAC1, the RAC1 specific antagonist NSC $23766(10 \mu \mathrm{M})$ was added $1 \mathrm{~h}$ prior to $\mathrm{H}_{2} \mathrm{O}_{2}$ exposure. The cell lysates were collected for GST-PAK1-PBD pulldown assay of RAC1 activation (GTP-bound RAC1 levels) and immunoblotting analysis of total RAC1. (B) Western blot analysis of phosphorylated MAP2K7 (p-MAP2K7) and total MAP2K7 in GCs with the indicated treatments as described above. TUBA1A served as the control for loading. (C) The detection of JNK activity in GCs subjected to the treatments as mentioned earlier. Data represent mean \pm S.E.; $n=3$. ${ }^{* *}$ Represents $P<0.01$ vs control group. ${ }^{\#}$ Represents $P<0.01$ vs $\mathrm{H}_{2} \mathrm{O}_{2}$ group. $\mathrm{N}$, not significant, $P>0.05$. (D) GCs transfected with scrambled control siRNA (SC siRNA) or Map $2 k 7$ siRNA were cultured with $10 \mu \mathrm{M}$ melatonin for $24 \mathrm{~h}$, washed in PBS, and then grown in medium containing $0.2 \mathrm{mM} \mathrm{H}_{2} \mathrm{O}_{2}$ for $2 \mathrm{~h}$. The expression of phosphorylated MAP2K7 (p-MAP2K7) and total MAP2K7 was determined by Western blotting. TUBA1A served as the control for loading. (E) GCs transfected with scrambled control siRNA (SC siRNA) or Map2k7 siRNA for $48 \mathrm{~h}$ were cultured with or without $0.2 \mathrm{mM} \mathrm{H}_{2} \mathrm{O}_{2}$ for $2 \mathrm{~h}$. Cell lysates were collected for Western blot analysis of RAC1 activation (GTP-RAC1 levels) by GST-PAK1-PBD pulldown and of total RAC1 levels. (F) GCs transfected with scrambled control siRNA (SC siRNA) or Map2k7 siRNA for $24 \mathrm{~h}$ were cultured for another $24 \mathrm{~h}$ in the presence or absence of $10 \mu \mathrm{M}$ melatonin before $2 \mathrm{~h}$ of $\mathrm{H}_{2} \mathrm{O}_{2}(0.2 \mathrm{mM})$ incubation. Cells were then processed for JNK determination. Data represent mean \pm S.E.; $n=3$. **Represents $P<0.01$ vs control group. ${ }^{* *}$ Represents $P<0.01$ vs $\mathrm{H}_{2} \mathrm{O}_{2}$ group. N, not significant, $P>0.05$. 
of JNK activity in GCs through the MAP2K7 pathway. We then examined the relationship between RAC1 and MAP2K7. It was found that blocking GTP-RAC1 formation with melatonin or NSC 23766 abrogated $\mathrm{H}_{2} \mathrm{O}_{2}$-induced MAP2K7 phosphorylation, whereas the RAC 1 antagonist provided no further inhibitory effect on MAP2 K7 activity in the presence of melatonin (Fig. 2A, B and Supplementary Fig. 1A, B). In contrast, MAP2K7 knockdown had little effect on $\mathrm{H}_{2} \mathrm{O}_{2}$-induced RAC1 activation (Fig. 2E and Supplementary Fig. 1D). Collectively, the RAC1/MAP2K7 pathway might be involved in melatonin-directed inhibition of JNK activation upon oxidative stimulation.

To determine whether the suppression of $\mathrm{H}_{2} \mathrm{O}_{2}$ triggered JNK activation by melatonin is a melatonin receptor-mediated event, we treated cells with Luzindole, a competitive antagonist of melatonin receptor. However, Luzindole did not significantly influence the inhibitory effects of melatonin on JNK activity upon $\mathrm{H}_{2} \mathrm{O}_{2}$ exposure (Supplementary Fig. 2).

To further assess whether JNK affected the prosurvival actions of melatonin under oxidative stress conditions, GCs were treated with JNK inhibitor SP600125. As shown in Fig. 1E, both melatonin and SP600125 displayed preventive effects on GC death upon $\mathrm{H}_{2} \mathrm{O}_{2}$ exposure. However, melatonin could not further restore the viability of cells pretreated with the JNK inhibitor. Based on these data, we proposed that the suppression of JNK is required for melatonin-mediated GC protection during oxidative stress.

\section{The suppression of JNK by melatonin protects GCs from oxidative stress-induced autophagic death}

Recent studies suggested that the antioxidative effect of melatonin is partly attributed to its role in autophagy regulation (Khaldy et al. 2003, Vega-Naredo et al. 2012). To test whether melatonin exerts any influence on autophagy in GCs with oxidative stress, we examined the levels of the phosphatidylethanolamine-conjugated microtubule-associated protein 1 light chain 3B-II (MAP1LC3B-II), which is a representative marker of autophagy (Klionsky et al. 2016). Immunoblotting results showed that treatment of melatonin for $24 \mathrm{~h}$ markedly reduced the accumulation of MAP1LC3B-II following $2 \mathrm{~h}$ of $\mathrm{H}_{2} \mathrm{O}_{2}(0.2 \mathrm{mM}$ ) exposure (Fig. $3 \mathrm{~A}$ and $\mathrm{B}$ ). Accordingly, it was accompanied by compromised degradation of SQSTM1, which functions as an adaptor protein for autophagy (Klionsky et al. 2016) (Fig. 3C). The inhibition of JNK with SP600125 also impaired $\mathrm{H}_{2} \mathrm{O}_{2}$-induced autophagy (Fig. $3 \mathrm{~A}, \mathrm{~B}$ and $\mathrm{C}$ ), but the inhibitor could not further restrain the autophagic response in cells pretreated with melatonin.

To obtain a better estimation of the cellular autophagic response to the melatonin-JNK signaling during oxidative stress, we next detected the autophagic flux by monitoring GCs transfected with GFP-MAP1LC3B- expressing vector (Fig. 3D and E). The numbers of green puncta, which represent autophagic vacuoles, were markedly increased in GCs subjected to $\mathrm{H}_{2} \mathrm{O}_{2}$ incubation. In contrast, both melatonin and SP600125 blocked the formation of autophagic punta upon oxidative stress. Moreover, no additional decline in autophagosome formation was observed in melatonintreated cells despite JNK inhibitor administration. The results indicated that melatonin-induced deactivation of JNK might contribute to the prohibition of autophagy in GCs during oxidative stress.

Growing evidence reveals a close correlation between autophagy and cell death under stressful conditions (Levine \& Kroemer 2008). To investigate whether the suppression of JNK-dependent autophagy is responsible for melatonin-mediated GC protection upon oxidative stress, cells were treated with melatonin, SP600125 or the autophagy inhibitor 3-methyladenine (3-MA) prior to $\mathrm{H}_{2} \mathrm{O}_{2}$ exposure. As shown in Fig. 3F, the cell viability loss during oxidative stress was reversed by the inhibition of autophagy and/or JNK. In accordance with this, melatonin displayed similar level of suppression in $\mathrm{H}_{2} \mathrm{O}_{2}$-induced cell death. Moreover, SP600125 and/or 3-MA did not significantly change cell viability in GCs treated with melatonin. In particular, the inhibition of autophagy failed to further improve GC survival following the co-treatment of melatonin and SP600125. Therefore, our data suggested that melatonin provides a preventive effect on oxidative stress-induced autophagic GC death by antagonizing the JNK signaling.

\section{Melatonin inhibits JNK-dependent upregulation of BECN1 expression during autophagy}

Considering the negative influence of melatonin on JNK function, we examined the expression of BCL-2 and BECN1, both of which belong to the downstream effectors of the JNK signaling (Wei et al. 2008). qRT-PCR analysis showed that Becn 1 mRNA levels were significantly increased upon oxidative stimulation, whereas both melatonin and SP600125 (JNK inhibitor) counteracted $\mathrm{H}_{2} \mathrm{O}_{2}$-induced transcriptional activation of Becn 1 (Fig. 4B). Notably, SP600125 provided no additional inhibitory effects on Becn 1 transcription in GCs pretreated with melatonin, indicating a potential downregulation of Becn 1 by melatonin through the JNK pathway (Fig. 4B). Western blotting results shown in Fig. $4 \mathrm{C}$ and $\mathrm{E}$ further confirmed that the melatoninmediated JNK deactivation contributes to the suppression of BECN1, a critical platform protein for autophagosome formation (Levine \& Yuan 2005). However, no evident induction of BCL-2 was observed under the same conditions (Fig. 4A, C and D). Nevertheless, these data suggested the possibility that the melatonin-JNK signaling might regulate autophagy with a BECN1dependent manner. 

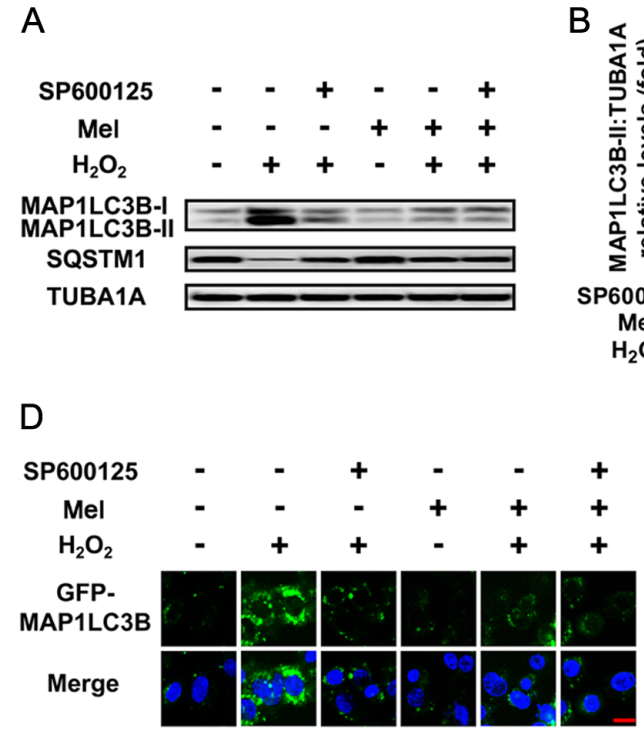

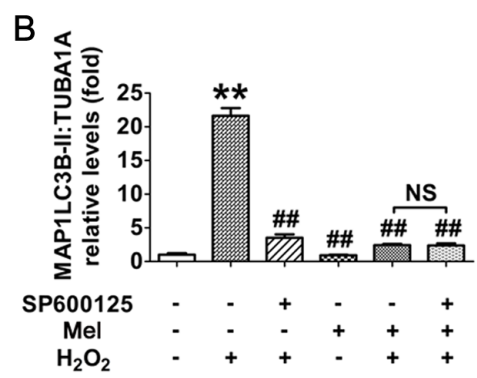

E

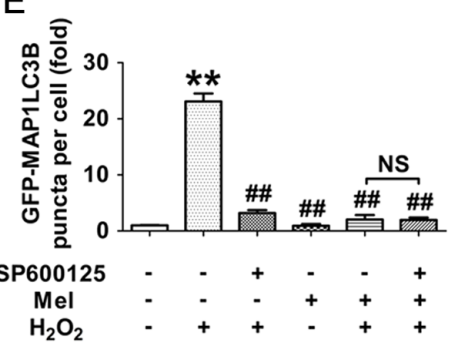

C

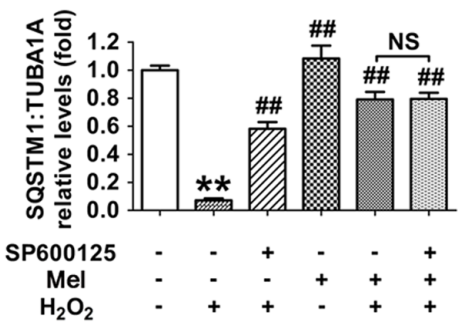

$\mathrm{F}$

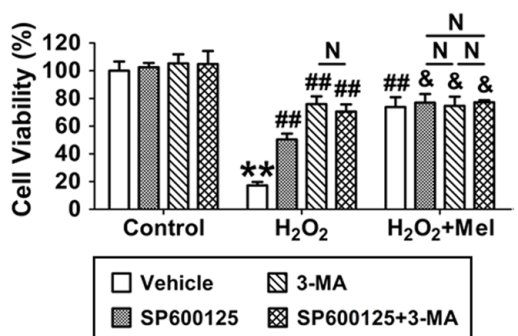

Figure 3 Melatonin prevents GCs from oxidative injury by repressing JNK-dependent autophagy. (A) After culturing with $10 \mu \mathrm{M}$ melatonin for $24 \mathrm{~h}$, GCs were rinsed in PBS and subjected to $2 \mathrm{~h}$ of $\mathrm{H}_{2} \mathrm{O}_{2}(0.2 \mathrm{mM})$ incubation. For the inhibition of JNK activity, SP600125 (25 $\left.\mu \mathrm{M}\right)$ was added $1 \mathrm{~h}$ before $\mathrm{H}_{2} \mathrm{O}_{2}$ treatment. The expression of MAP1LC3B and SQSTM1 in GCs was determined by Western blotting. (B and C) Quantification of MAP1LC3B-II accumulation and SQSTM1 degradation using densitometric analysis. TUBA1A served as the control for loading. Data represent mean \pm s.E.; $n=3$. **Represents $P<0.01$ vs control group. ${ }^{\sharp}$ Represents $P<0.01 \mathrm{vs}_{2} \mathrm{O}_{2}$ group. NS, not significant, $P>0.05$. (D) GCs transfected with GFP-MAP1LC3B plasmid for $24 \mathrm{~h}$ were grown with or without $10 \mu \mathrm{M}$ melatonin for another $24 \mathrm{~h}$ before $2 \mathrm{~h}$ of $\mathrm{H}_{2} \mathrm{O}_{2}(0.2 \mathrm{mM})$ incubation. SP600125 $(25 \mu \mathrm{M})$ was added $1 \mathrm{~h}$ prior to $\mathrm{H}_{2} \mathrm{O}_{2}$ exposure. Laser confocal-scanning microscopy was employed to observe the GFP fluorescent puncta in GCs. Bar, $10 \mu \mathrm{m}$. (E) Quantification of the punctate GFP-MAP1LC3B per cell. Experiments were repeated in triplicate, and three fields

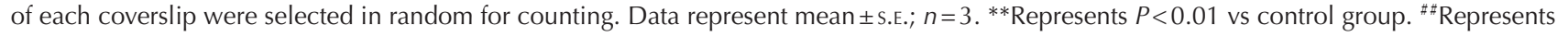
$P<0.01$ vs $\mathrm{H}_{2} \mathrm{O}_{2}$ group. NS, not significant, $P>0.05$. (F) GCs with $24 \mathrm{~h}$ of melatonin $(10 \mu \mathrm{M})$ treatment were rinsed in PBS, and then exposed to $\mathrm{H}_{2} \mathrm{O}_{2}(0.2 \mathrm{mM})$ for $2 \mathrm{~h}$. The autophagy inhibitor 3-MA (10 mM) or the JNK inhibitor SP600125 $(25 \mu \mathrm{M})$ was added $1 \mathrm{~h}$ before $\mathrm{H}_{2} \mathrm{O}_{2}$ incubation. Cell viability was determined using the CCK-8 assay. Data represent mean \pm S.E.; $n=3$ in each group. ${ }^{* *}$ Represents $P<0.01$ compared to non-treatment control group. ${ }^{\sharp}$ Represents $P<0.01$ compared to $\mathrm{H}_{2} \mathrm{O}_{2}$-only-treated cells. ${ }^{\text {\& }}$ Represents $P>0.05$ compared to $\mathrm{H}_{2} \mathrm{O}_{2}+$ melatonin group. $\mathrm{N}$, not significant, $P>0.05$.

\section{Melatonin-mediated JNK inhibition facilitates the formation of BCL-2/BECN1 complex}

Since the activation of JNK has been reported to promote the dissociation between BCL-2 and BECN1 (Wei et al. 2008), which is required for autophagy induction under stressful conditions (Pattingre et al. 2005), we wonder whether melatonin-induced JNK suppression affects the interactions between BCL-2 and BECN1 in GCs suffering oxidative damage. To test this assumption, GCs exposed to $\mathrm{H}_{2} \mathrm{O}_{2}$ incubation following melatonin and/or SP600125 treatment were collected for immunoprecipitation with the antibody against BCL-2. As shown in Fig. 5A, similar levels of BCL-2 expression
A

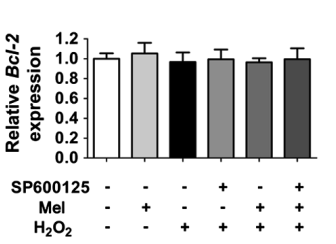

B

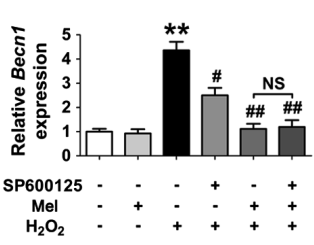

C

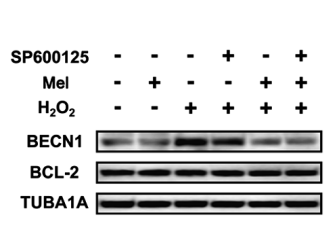

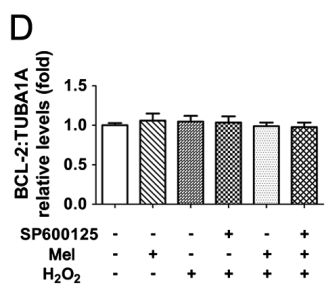

$\mathrm{E}$

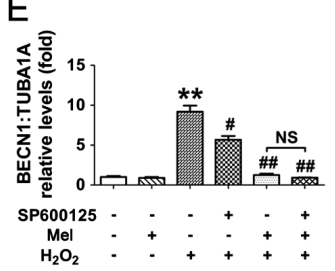

Figure 4 Suppression of JNK by melatonin reduces BECN1 expression during autophagy. (A and B) Primary cultured GCs with $24 \mathrm{~h}$ of melatonin $(10 \mu \mathrm{M})$ pretreatment were rinsed in PBS and stimulated with $\mathrm{H}_{2} \mathrm{O}_{2}(0.2 \mathrm{mM})$ for $2 \mathrm{~h}$. The JNK inhibitor SP600125 $(25 \mu \mathrm{M})$ were added into the culture medium $1 \mathrm{~h}$ before $\mathrm{H}_{2} \mathrm{O}_{2}$ exposure. The mRNA expression of BCl-2 (A) and Becn 1 (B) in GCs was determined by qRT-PCR. Expression data were normalized to that of $A c t b$. Data represent mean \pm S.E.; $n=3$. ${ }^{* *}$ Represents $P<0.01$ compared to control group. ${ }^{*}$ Represents $P<0.05$ compared to $\mathrm{H}_{2} \mathrm{O}_{2}$ group. ${ }^{\#}$ Represents $P<0.01$ compared to $\mathrm{H}_{2} \mathrm{O}_{2}$ group. NS, not significant, $P>0.05$. (C) Immunoblotting analysis of BCL-2 and BECN1 in GCs with the indicated treatments as described earlier. (D and E) Bar charts show the quantification of endogenous BCL-2 and BECN1. TUBA1A served as the control for loading. Data represent mean \pm s.E.; $n=3$. **Represents $P<0.01$ compared to control group.

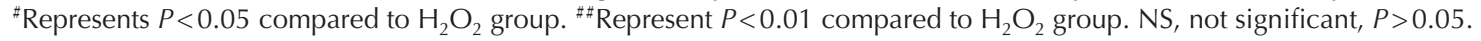


were detected in the whole-cell lysates harvested from each group. When the corresponding IP components were immunostained using anti-BCL-2, a single band of about $26 \mathrm{kDa}$ was observed, indicating that BCL-2 was successfully immunoprecipitated (Fig. 5A and B). Western blot analysis of the IP contents revealed that BECN1 was coprecipitated by the BCL-2 antibody (Fig. 5A), implying a physical interaction between BCL-2 and BECN1. However, the binding affinity of BECN1 to $\mathrm{BCL}-2$ was markedly repressed upon oxidative stimulation (Fig. 5A and C). In contrast, both melatonin and SP600125 partly restored the formation of BCL-2/ BECN1 complex upon $\mathrm{H}_{2} \mathrm{O}_{2}$ exposure (Fig. 5A and C). Importantly, the JNK inhibitor failed to further improve BECN1-BCL-2 interaction in cells pretreated with melatonin (Fig. 5A and C). Therefore, the formation of BCL-2/BECN1 complex might be an essential step in melatonin-mediated suppression of autophagy through the JNK signaling pathway.

\section{The protective effect of melatonin on GC survival by inhibiting JNK-induced autophagy is independent of ROS scavenging}

Melatonin can protect cells directly as an antioxidant or indirectly through activation of several antioxidative components, including superoxide dismutase (SOD), catalase (CAT), glutathione peroxidase (GPx), glutathione reductase (GR) and glutathione (GSH) (Shen et al. 2014). Our current treatment procedure eliminated the direct antioxidative action of melatonin, because GCs incubated with this indoleamine for $24 \mathrm{~h}$ were rinsed in PBS before $\mathrm{H}_{2} \mathrm{O}_{2}$ exposure. To verify whether the prosurvival role of melatonin-JNK signaling is achieved by removing oxidative stress, cells were treated with specific antagonists targeted to the downstream antioxidants (hereafter referred as antioxidant inhibitors, or AOI) of melatonin. The results of ROS detection showed that AOI abolished melatoninmediated inhibition of oxidative stress despite SP600125 administration (Fig. 6A and B), indicating that JNK is not necessary for the antioxidation capacity of melatonin. However, AOI did not significantly influence the inhibitory effects of melatonin on GC death (Fig. 6C) or JNK activity (Supplementary Fig. 3) upon oxidative stimulation. Additionally, after AOI and SP600125 treatment, no apparent changes in cell viability were observed in GCs subjected to melatonin incubation during oxidative stress. Moreover, when cells were treated with both melatonin and AOI, the JNK inhibitor could not further improve GC resistance to oxidative damage. These findings thus suggested a potential role of melatonin-JNK signaling in preventing oxidative damage without eliminating oxidative stress itself.

We next investigated whether a nonspecific effect of melatonin might influence autophagy by antagonizing oxidative stress. By determining the autophagic flux
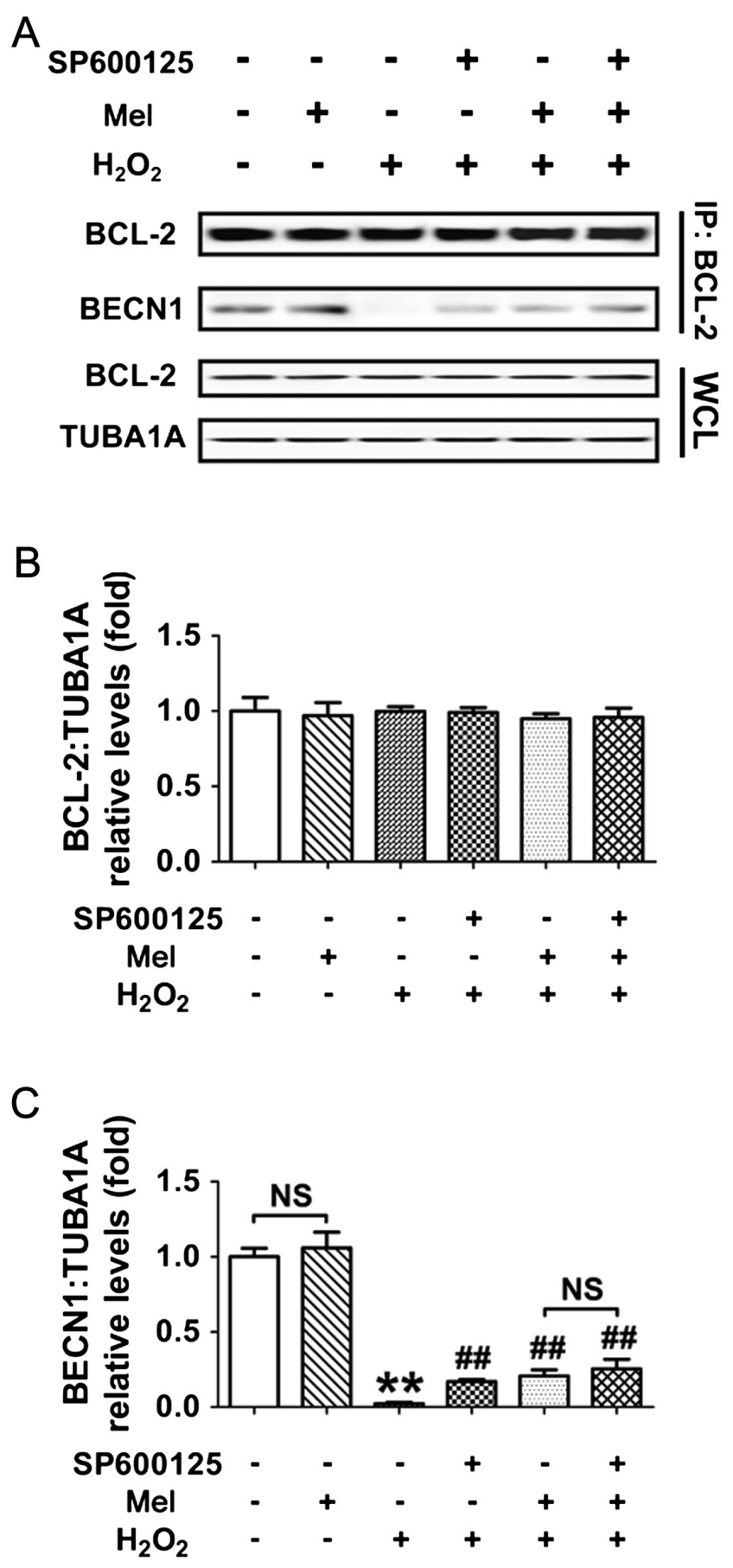

Figure 5 Melatonin maintains the association of BCL-2 and BECN1 by inhibiting JNK in $\mathrm{H}_{2} \mathrm{O}_{2}$-treated GCs. (A) Primary cultured GCs pretreated with or without $10 \mu \mathrm{M}$ melatonin were washed in PBS, and then grown in medium containing $0.2 \mathrm{mM} \mathrm{H}_{2} \mathrm{O}_{2}$ for $2 \mathrm{~h}$. To block the JNK signaling, SP600125 $(25 \mu \mathrm{M})$ was added $1 \mathrm{~h}$ before $\mathrm{H}_{2} \mathrm{O}_{2}$ incubation. After the indicated treatment, cell lysates were collected for co-immunoprecipitation with anti-BCL-2, followed by probing with anti-BECN1. WCL, whole-cell lysates. IP, immunoprecipitation. (B and $\mathrm{C}$ ) The amount of Co-immunoprecipitated BCL-2 (B) and BECN1 (C) was normalized to TUBA1A content in the whole-cell lysates (input). Data represent mean \pm S.E.; $n=3$ in each group. ${ }^{* *} P<0.01$ vs control group; ${ }^{\# \#} P<0.01$ vs $\mathrm{H}_{2} \mathrm{O}_{2}$ group. NS, not significant, $P>0.05$. 
A

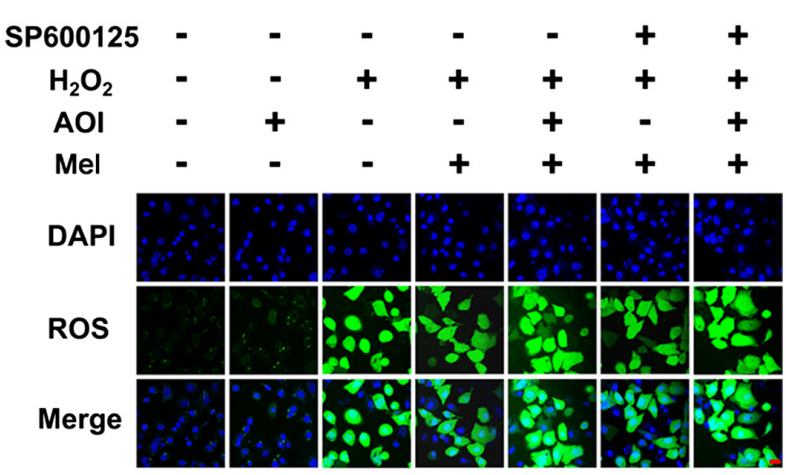

B

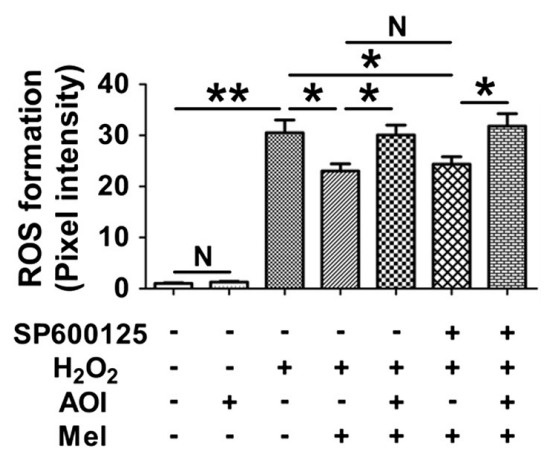

C

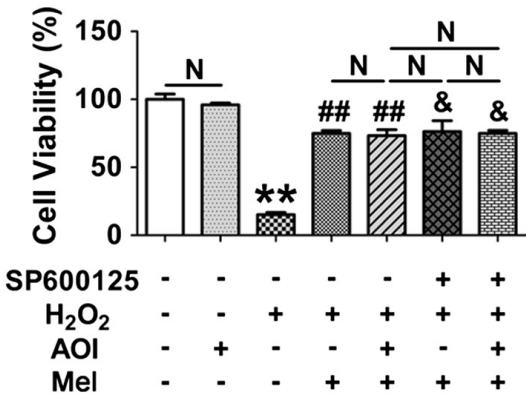

Figure 6 The melatonin-JNK signaling directly attenuates oxidative injury in GCs. (A) GCs grown in medium containing $10 \mu \mathrm{M}$ melatonin for $24 \mathrm{~h}$ were then rinsed in PBS and exposed to $2 \mathrm{~h}$ of $\mathrm{H}_{2} \mathrm{O}_{2}(0.2 \mathrm{mM})$ incubation. The JNK antagonist SP600125 $(25 \mu \mathrm{M})$ and inhibitors of melatonin downstream antioxidants (AOI) were added $1 \mathrm{~h}$ prior to $\mathrm{H}_{2} \mathrm{O}_{2}$ treatment. The ROS levels were detected by dichlorofluorescein fluorescence (green), and nuclei were counterstained with DAPI (blue). Bar, $20 \mu \mathrm{m}$. (B) The optical density of intracellular ROS was quantified using ImageJ software. Experiments were repeated in triplicate, and three fields of each coverslip were selected in random for counting. Data represent mean \pm S.E.; $n=3 .{ }^{*} P<0.05,{ }^{* *} P<0.01$; $\mathrm{N}$, not significant, $P>0.05$. (C) The measurement of cell viability by CCK-8 assay. GCs were treated as above. Data represent mean \pm S.E.; $n=3$ in each group. ${ }^{* *}$ Represents $P<0.01$ compared to non-treatment

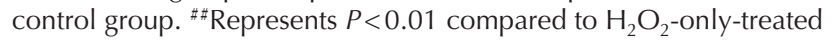
cells. ${ }^{\text {\&Represents }} P>0.05$ compared to $\mathrm{H}_{2} \mathrm{O}_{2}+$ melatonin group. $\mathrm{N}$, not significant, $P>0.05$.

in GCs transfected with GFP-MAP1LC3B plasmid, we found that $\mathrm{AOI}$ exerts no evident influence on melatonin-induced suppression of autophagosome

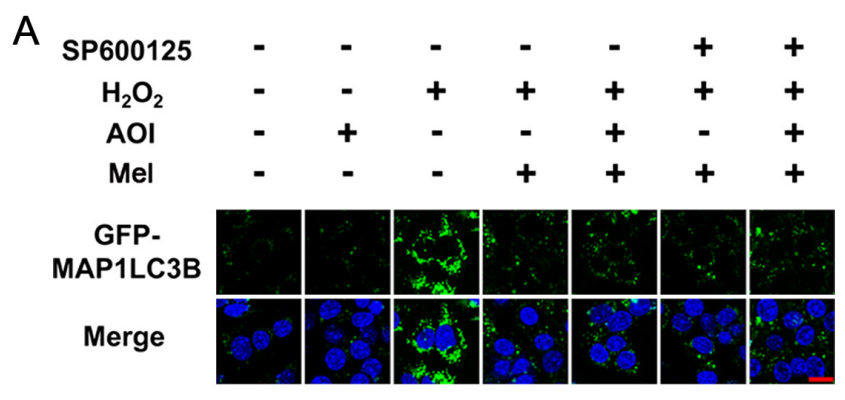

B

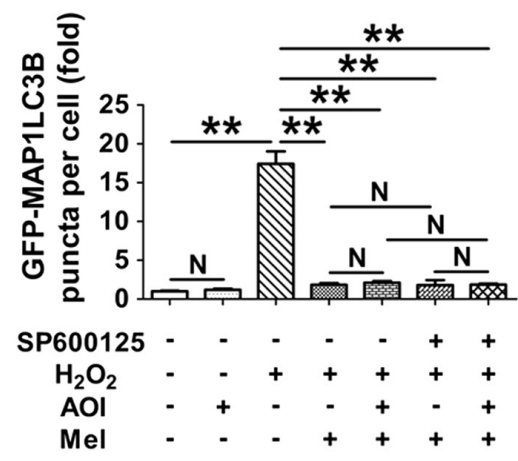

C
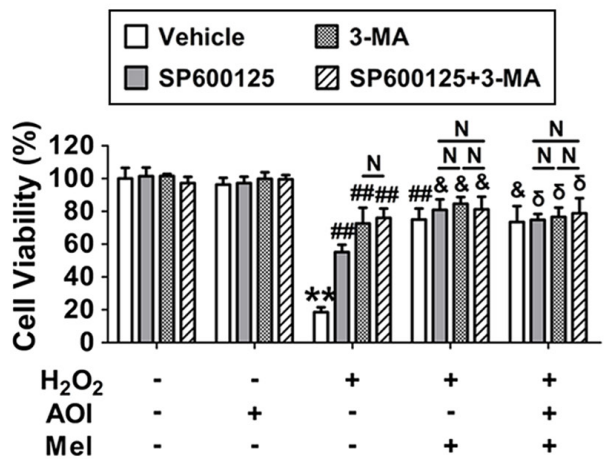

Figure 7 The inhibitory effect of melatonin on JNK-dependent autophagic GC death is independent of ROS elimination. (A) GCs transfected with GFP-MAP1LC3B plasmid for $24 \mathrm{~h}$ were cultured with or without $10 \mu \mathrm{M}$ melatonin for another $24 \mathrm{~h}$ before $2 \mathrm{~h}$ of $\mathrm{H}_{2} \mathrm{O}_{2}$ $(0.2 \mathrm{mM})$ incubation. The JNK antagonist SP600125 $(25 \mu \mathrm{M})$ and inhibitors of melatonin downstream antioxidants (AOI) were added $1 \mathrm{~h}$ before $\mathrm{H}_{2} \mathrm{O}_{2}$ exposure. Formation of GFP-MAP1LC3B vacuoles (puncta) was determined in GCs as described above. Bar, $10 \mu \mathrm{m}$. (B) Quantification of GFP-MAP1LC3B puncta per cell. Experiments were repeated in triplicate, and 3 fields of each coverslip were selected in random for counting. Data represent mean \pm S.E.; $n=3$. ${ }^{* *} P<0.01 ; \mathrm{N}$, not significant, $P>0.05$. (C) GCs with $24 \mathrm{~h}$ of melatonin $(10 \mu \mathrm{M})$ treatment were rinsed in PBS, and then subjected to $2 \mathrm{~h}$ of $\mathrm{H}_{2} \mathrm{O}_{2}(0.2 \mathrm{mM})$ exposure. The inhibitors against JNK (SP600125), autophagy (3-MA) or melatonin downstream antioxidants (AOI) were added into the culture medium $1 \mathrm{~h}$ before $\mathrm{H}_{2} \mathrm{O}_{2}$ incubation. Cell viability was examined as described above. **Represents $P<0.01$ compared to non treatment control group. \# Represents $P<0.01$ compared to $\mathrm{H}_{2} \mathrm{O}_{2}$-only-treated cells. \& Represents $P>0.05$ compared to $\mathrm{H}_{2} \mathrm{O}_{2}$ + melatonin group. ${ }^{\delta}$ Represents $P>0.05$ compared to $\mathrm{H}_{2} \mathrm{O}_{2}+\mathrm{AOI}+$ melatonin group. $\mathrm{N}$, not significant, $P>0.05$. 
formation in cells with $\mathrm{H}_{2} \mathrm{O}_{2}$ exposure (Fig. 7A and B). In particular, when GCs were pretreated with melatonin and $\mathrm{AOI}$, the inhibition of JNK provided no additional preventive effects on autophagy activation (Fig. 7A and $\mathrm{B}$ ). Correspondingly, loss of cell viability induced by $\mathrm{H}_{2} \mathrm{O}_{2}$ incubation was markedly reduced following melatonin, SP600125 and/or 3-MA treatment, but AOI did not seem to affect this process (Fig. 7C). Moreover, inhibitors of autophagy and/or JNK could not further restore cell viability following the treatment of melatonin and $\mathrm{AOI}$ (Fig. 7C). On the other hand, when JNK was blocked in cells treated with melatonin and AOI, the autophagy inhibitor 3-MA also failed to provide a greater protection to GCs upon oxidative stimulation (Fig. 7C). Collectively, these data further confirmed that the melatonin-JNK signaling directly inhibits autophagic death in GCs suffering oxidative damage.

\section{Discussion}

In response to metabolic or environmental stimuli, an excess production or decreased scavenging of reactive oxygen species (ROS) destroys cellular lipids, carbohydrates, proteins and DNA in a situation termed as oxidative stress. The adverse effects of oxidative stress on female reproduction have been well documented (Agarwal et al. 2012). For example, exposure to ionizing radiation, tobacco smoke or alcohol causes GC death and subsequent follicle loss in the ovary, leading to the pathogenesis of anovulatory infertility (Agarwal et al. 2012). Moreover, recent evidence reveals a potential correlation between GC autophagy and follicular degeneration in mice with cigarette smoke exposure (Gannon et al. 2012, 2013, Furlong et al. 2015). Melatonin, an indoleamine with antioxidant properties, has been implicated in preserving normal functions of GCs and follicles during oxidative stress (Tamura et al. 2009); however, the underlying mechanisms were still largely undetermined. In this study, we show for the first time that melatonin relieved oxidative damage in ovarian GCs by inhibiting JNK-mediated autophagy.

The present findings demonstrate that melatonin (1) prevents GC injury by restraining JNK activity, (2) inhibits JNK-dependent autophagy, (3) decreases autophagic GCs death by antagonizing the JNK pathway, (4) counteracts JNK-induced dissociation of BCL-2/BECN1 complex during the autophagic process and (5) provides an antioxidation-independent GC protection by targeting the JNK-autophagy signaling under oxidative stress conditions. Taken together, suppressing autophagy through the melatonin/JNK/BCL-2/BECN1 axis might constitute part of a survival mechanism for maintaining GC viability upon oxidative stimulation.

Melatonin is ubiquitously distributed in every bodily compartment including in follicular fluid. The physiological melatonin concentrations measured in follicular fluid are almost three times higher than that measured in the blood $(36.5 \pm 4.8 \mathrm{pg} / \mathrm{mL}$ vs $10.0 \pm 1.4 \mathrm{pg} /$ $\mathrm{mL}$ ) (Brzezinski et al. 1987). It is possible that melatonin may be produced by GCs (Tamura et al. 2009). However, it is currently believed that the bulk of melatonin detected in the ovary, and follicular fluid is derived from the circulation (Tamura et al. 2009). The presence of high melatonin levels in follicular fluid and the presence of melatonin receptors in GCs (Lee et al. 2001, Soares et al. 2003a) suggest a potential beneficial property of this indoleamine on ovarian function. Actually, melatonin has been implicated in preventing the pathogenesis of polycystic ovary syndrome (PCOS) and premature ovarian failure (POF) (Tamura et al. 2009). In contrast, low intrafollicular melatonin concentrations in patients with PCOS were associated with anovulation and poor oocyte quality (Tamura et al. 2009). In the present study, data obtained from cultured GCs demonstrate a potential role for melatonin in protecting against autophagic GC death upon oxidative stress. Therefore, melatonin might be beneficial for the therapy for stressrelated anovulatory infertility, although more in vivo experiments should be performed to investigate the underlying mechanism of melatonin for maintaining the well-being of GCs and ovarian follicles in future studies.

The c-Jun N-terminal kinase (JNK) branch of the mitogen-activated protein plays a critical role in cellular responses to mitogenic stimuli and environmental stresses (Weston \& Davis 2007). Persistent JNK activation by UV radiation or $\mathrm{H}_{2} \mathrm{O}_{2}$ exposure has been reported to induce cell death (Vallyathan 2002). However, there is no definite evidence about what response JNK will take when melatonin-treated ovarian GCs experience oxidative stress. To determine the mechanism by which melatonin protects GCs from oxidative injury, the effect of JNK on cell death was investigated. Here, we found that $\mathrm{H}_{2} \mathrm{O}_{2}$ incubation improved JNK activation as suggested by increased cellular conversion of $\mathrm{NADH}$ to NAD, which was associated with elevated ROS production upon oxidative stress (Fig. 1). In contrast, melatonin exhibited an inhibitory effect on JNK activation during oxidative stimulation (Fig. 1). The protein kinases RAC1 and MAP2K7 have been implicated in cellular responses to various types of external stimuli (Moriguchi et al. 1997, Ozaki et al. 2000). In this study, it was found that both RAC1 and MAP2K7 were activated by $\mathrm{H}_{2} \mathrm{O}_{2}$ in GCs to promote JNK activation, but all three kinases were inhibited when cells were pretreated with melatonin before $\mathrm{H}_{2} \mathrm{O}_{2}$ exposure (Fig. 2). Further experiments using antagonists against RAC1, MAP2K7 or JNK identified RAC1 as an upstream activator of MAP2K7, which in turn stimulated the activation of JNK during oxidative stress. Conversely, melatonin might repress $\mathrm{H}_{2} \mathrm{O}_{2}$ induced JNK activation through the RAC1/MAP2K7 pathway (Fig. 2). We also tested the possible regulation of melatonin on JNK activity by examining the direct action of melatonin as an antioxidant to scavenge free radicals (Supplementary Fig. 3) or indirect action of melatonin 
to bind its receptors (Supplementary Fig. 2). However, no definitive evidence was observed for the involvement of either the direct or indirect actions of melatonin in preventing oxidative stress-triggered JNK activation. Therefore, RAC1/MAP2K7 signaling might be one of the major pathways required for melatonin-mediated inhibition of JNK activation upon oxidative stimulation. We then determined whether JNK exerts any influence on the prosurvival effects of melatonin under oxidative stress conditions. Notably, JNK serves as a critical mediator of $\mathrm{H}_{2} \mathrm{O}_{2}$-induced $\mathrm{GC}$ death, since the decline in cell viability was reversed in the presence of JNK inhibitor SP600125 (Fig. 1). Importantly, melatonin exhibited an inhibitory effect on both JNK activity and GC death; however, melatonin provided no additional protection to GCs when JNK was blocked (Fig. 1). Collectively, these data first demonstrate that JNK suppression is required for melatonin-mediated GC protection.

As a stress-activated protein kinase, JNK has been linked to autophagy induction upon oxidative stimulation (Zhou et al. 2015). Earlier evidence from neuronal cells suggested that ROS accumulation resulting from neurotoxin treatment led to JNK-induced activation of autophagy (Rodriguez-Blanco et al. 2012), whereas inhibitors of JNK and its downstream substrates antagonized the autophagic response in the hippocampus of mice (Borsello et al. 2003). Oxidative stress has been shown to induce autophagy in ovarian GCs (Shen et al. 2016, 2017), but it remains elusive whether JNK plays a role in this process. On the other hand, growing evidence in recent years indicates a potential function of melatonin in autophagy regulation during oxidative stress (Coto-Montes et al. 2012). Particularly, melatonin may serve primarily as a mitigator when autophagy is over-stimulated (Coto-Montes et al. 2012). The current study showed that both JNK inhibitor SP600125 and melatonin inhibited the autophagic flux in $\mathrm{H}_{2} \mathrm{O}_{2}$ incubated GCs, but SP600125 could not further repress autophagy when cells were pretreated with melatonin. Thus, melatonin may suppress oxidative stress-induced autophagy in GCs though a JNK-dependent manner.

Autophagy is a process of catabolism that controls cell homeostasis. Basal level of autophagy acts as a cytoprotective mechanism through orderly clearance and recycling of dysfunctional cytoplasmic components, but autophagy may also culminate into a cell death modality when the intracellular constituents are excessively degraded (Tsujimoto \& Shimizu 2005, Levine \& Kroemer 2008). Research on acute toxic neuronal death showed that autophagic PCD (programmed cell death) induced by $\mathrm{N}$-methyl-d-aspartate treatment was accompanied with JNK pathway activation, c-Jun-selective phosphorylation and c-Fos-specific upregulation in hippocampal neurons. By contrast, the induction of autophagy was markedly repressed by JNK antagonist, indicating that the autophagic death of neuronal cells observed here was regulated by JNK pathway (Borsello et al. 2003). Considering the role of melatonin in preventing JNK-dependent autophagy as mentioned earlier, we examined the interplay between autophagy, JNK and cell death in $\mathrm{H}_{2} \mathrm{O}_{2}$-treated GCs following melatonin administration. To our knowledge, this is the first evidence demonstrating that the melatoninmediated JNK suppression prevents a detrimental form of autophagy in GCs during oxidative stress.

There have been multiple lines of evidence suggesting that the anti-apoptotic protein $\mathrm{BCL}-2$ negatively regulates autophagy by blocking BECN1, an essential component of the autophagy initiation complex (Pattingre et al. 2009). BECN1 serves to recruit autophagy/endosomal regulatory proteins, such as PIK3C3, UVRAG and ATG14, all of which are required for autophagosome biogenesis (Levine \& Yuan 2005). BECN1 also binds to BCL-2 family members (Erlich et al. 2007). This binding decreases the association of PIK3C3 with BECN1, and thereby inhibiting the autophagic process (Maiuri et al. 2007). Here, we found that autophagy is correlated with the BCL-2/BECN1 system in GCs suffering oxidative stress. $\mathrm{H}_{2} \mathrm{O}_{2}$ incubation upregulated $\mathrm{BECN} 1$ expression and increased the level of free form BECN1, which had been detached from BCL-2/ BECN1 complex. In contrast, JNK inhibitor (SP600125) enhanced the interaction between BCL-2 and BECN1. The results are supported by a previous report (Park et al. 2009), which showed that the elevated expression of BECN1 and its dissociation from BCL-2 are responsible for JNK-mediated autophagic cell death. Notably, melatonin also enhanced the interaction between BCL-2 and BECN1, but SP600125 did not further restore the formation of BCL-2/BECN1 complex in the presence of melatonin. These data suggest that BCL-2/BECN1 disassembly occurs in oxidative stress-induced GC injury, whereas melatonin attenuates this process via suppressing JNK. However, the exact downstream signaling of melatonin-JNK pathway in preventing oxidative injury via autophagy is yet to be investigated in GCs.

Since the BCL-2/BECN1 complex regulates both apoptosis and autophagy by modulating of the counterbalance between cell survival and cell death (Pattingre et al. 2005), a possible interaction between apoptotic cell death and autophagic PCD might exist in melatonin-mediated GC protection upon oxidative stimulation. However, our earlier reports showed that no evident apoptotic signals occur in the early stage of oxidative stress-induced GCs damage (Shen et al. 2016, 2017). Further investigations revealed that autophagic PCD represents a major form of GC death, which is independent of apoptosis in GCs suffering short-term (2 $\mathrm{h}$ of $\mathrm{H}_{2} \mathrm{O}_{2}$ exposure) oxidative stress (Shen et al. 2016, 2017). Therefore, apoptosis may not be active in our current model. Nevertheless, this situation enables us to specify the effects of autophagic PCD without the interference from apoptosis.

In summary, our findings showed excessive activation of autophagy induced by oxidative stress through the JNK 
pathway in GCs. The detrimental effects of autophagic induction could be counteracted by melatonin-mediated JNK suppression, which protected GCs from oxidative damage. Moreover, we demonstrated a potential role for melatonin in protecting against autophagic GC death via the JNK/BCL-2/BECN1 signaling axis. Therefore, targeting this pathway using small molecular inhibitors may provide novel therapy for stress-related anovulatory infertility.

\section{Supplementary data}

This is linked to the online version of the paper at https://doi.org/10.1530/REP-18-0002.

\section{Declaration of interest}

The authors declare that there is no conflict of interest that could be perceived as prejudicing the impartiality of the research reported.

\section{Funding}

This work was supported by the National Natural Science Foundation of China (No. 31601939), the Fundamental Research Funds for the Central Universities (No. KJQN201705), Natural Science Foundation of Jiangsu Province (No. BK20150664), Key Program of National Natural Science Foundation of China (No. 31630072), the key Project of Chinese National Programs for Fundamental Research and Development (973 program no. 2014CB138502), China Postdoctoral Science Special Foundation (No. 2016T90476), China Postdoctoral Science Foundation (No. 2015M581818) and Postdoctoral Fund in Jiangsu Province (No. 1501047A).

\section{Author contribution statement}

M S conceived the project. M S designed the study. M S and $H L L$ supervised the entire research. M S and $Y C$ performed the experiments. Y J contributed to some technical supports. M S, H L L and S-C S discussed the study. M S contributed to data analysis, figure preparation and manuscript writing. All the authors reviewed the manuscript.

\section{Acknowledgments}

The authors thank Prof. Jiyong Zhou (Zhejiang University) for provision of the green fluorescent protein (GFP)tagged microtubule-associated protein 1 light chain 3 beta (MAP1LC3B) expression plasmid. Co-authors: Yan Cao, Yi Jiang, Shao-chen Sun.

\section{References}

Agarwal A, Aponte-Mellado A, Premkumar BJ, Shaman A \& Gupta S 2012 The effects of oxidative stress on female reproduction: a review. Reproductive Biology and Endocrinology 10 49. (https://doi. org/10.1186/1477-7827-10-49)

Birben E, Sahiner UM, Sackesen C, Erzurum S \& Kalayci O 2012 Oxidative stress and antioxidant defense. World Allergy Organization Journal 5 9-19. (https://doi.org/10.1097/WOX.0b013e3182439613)

Borsello T, Croquelois K, Hornung JP \& Clarke PG 2003 N-methyl-daspartate-triggered neuronal death in organotypic hippocampal cultures is endocytic, autophagic and mediated by the c-Jun $\mathrm{N}$-terminal kinase pathway. European Journal of Neuroscience 18 473-485. (https://doi. org/10.1046/j.1460-9568.2003.02757.x)

Brzezinski A, Seibel MM, Lynch HJ, Deng MH \& Wurtman RJ 1987 Melatonin in human preovulatory follicular fluid. Journal of Clinical Endocrinology and Metabolism 64 865-867. (https://doi.org/10.1210/ jcem-64-4-865)

Coto-Montes A, Boga JA, Rosales-Corral S, Fuentes-Broto L, Tan DX \& Reiter RJ 2012 Role of melatonin in the regulation of autophagy and mitophagy: a review. Molecular and Cellular Endocrinology 361 12-23. (https://doi.org/10.1016/j.mce.2012.04.009)

Duerrschmidt N, Zabirnyk O, Nowicki M, Ricken A, Hmeidan FA, Blumenauer V, Borlak J \& Spanel-Borowski K 2006 Lectin-like oxidized low-density lipoprotein receptor-1-mediated autophagy in human granulosa cells as an alternative of programmed cell death. Endocrinology 147 3851-3860. (https://doi.org/10.1210/en.20060088)

Erlich S, Mizrachy L, Segev O, Lindenboim L, Zmira O, Adi-Harel S, Hirsch JA, Stein R \& Pinkas-Kramarski R 2007 Differential interactions between Beclin 1 and Bcl-2 family members. Autophagy 3 561-568. (https://doi.org/10.4161/auto.4713)

Furlong HC, Stampfli MR, Gannon AM \& Foster WG 2015 Cigarette smoke exposure triggers the autophagic cascade via activation of the AMPK pathway in mice. Biology of Reproduction 93 93. (https://doi. org/10.1095/biolreprod.115.132183)

Gannon AM, Stampfli MR \& Foster WG 2012 Cigarette smoke exposure leads to follicle loss via an alternative ovarian cell death pathway in a mouse model. Toxicological Sciences 125 274-284. (https://doi. org/10.1093/toxsci/kfr279)

Gannon AM, Stampfli MR \& Foster WG 2013 Cigarette smoke exposure elicits increased autophagy and dysregulation of mitochondrial dynamics in murine granulosa cells. Biology of Reproduction 88 63. (https://doi. org/10.1095/biolreprod.112.106617)

Gupta RK, Miller KP, Babus JK \& Flaws JA 2006 Methoxychlor inhibits growth and induces atresia of antral follicles through an oxidative stress pathway. Toxicological Sciences 93 382-389. (https://doi.org/10.1093/ toxsci/kfl052)

Gustafsson AB \& Gottlieb RA 2009 Autophagy in ischemic heart disease. Circulation Research 104 150-158. (https://doi.org/10.1161/ CIRCRESAHA.108.187427)

Kaipia A \& Hsueh AJ 1997 Regulation of ovarian follicle atresia. Annual Review of Physiology 59 349-363. (https://doi.org/10.1146/annurev. physiol.59.1.349)

Khaldy H, Escames G, Leon J, Bikjdaouene L \& Acuna-Castroviejo D 2003 Synergistic effects of melatonin and deprenyl against MPTPinduced mitochondrial damage and DA depletion. Neurobiology of Aging 24 491-500. (https://doi.org/10.1016/S0197-4580(02) 00133-1)

Klionsky DJ, Abdelmohsen K, Abe A, Abedin MJ, Abeliovich H, Acevedo Arozena A, Adachi H, Adams CM, Adams PD, Adeli K et al. 2016 Guidelines for the use and interpretation of assays for monitoring autophagy (3rd edition). Autophagy 12 1-222. (https://doi.org/10.1080/ 15548627.2015.1100356)

Kroemer G \& Levine B 2008 Autophagic cell death: the story of a misnomer. Nature Reviews Molecular Cell Biology 9 1004-1010. (https://doi. org/10.1038/nrm2529)

Lee CJ, Do BR, Lee YH, Park JH, Kim SJ, Kim JK, Roh SI, Yoon YD \& Yoon HS 2001 Ovarian expression of melatonin Mel(1a) receptor mRNA during mouse development. Molecular Reproduction and Development 59 126-132. (https://doi.org/10.1002/mrd.1015)

Levine B \& Klionsky DJ 2004 Development by self-digestion: molecular mechanisms and biological functions of autophagy. Developmental Cell 6 463-477. (https://doi.org/10.1016/S1534-5807(04)00099-1)

Levine B \& Kroemer G 2008 Autophagy in the pathogenesis of disease. Cell 132 27-42. (https://doi.org/10.1016/j.cell.2007.12.018) 
Levine B \& Yuan J 2005 Autophagy in cell death: an innocent convict? Journal of Clinical Investigation 115 2679-2688. (https://doi.org/10.1172/ JCl26390)

Liang R, Nickkholgh A, Hoffmann K, Kern M, Schneider H, Sobirey M, Zorn M, Buchler MW \& Schemmer P 2009 Melatonin protects from hepatic reperfusion injury through inhibition of IKK and JNK pathways and modification of cell proliferation. Journal of Pineal Research $\mathbf{4 6}$ 8-14. (https://doi.org/10.1111/j.1600-079X.2008.00596.x)

Maiuri MC, Le Toumelin G, Criollo A, Rain JC, Gautier F, Juin P, Tasdemir E, Pierron G, Troulinaki K, Tavernarakis N et al. 2007 Functional and physical interaction between $\mathrm{BCl}-\mathrm{X}(\mathrm{L})$ and a $\mathrm{BH} 3$-like domain in Beclin-1. EMBO Journal 26 2527-2539. (https://doi.org/10.1038/sj.emboj.7601689)

Manchester LC, Coto-Montes A, Boga JA, Andersen LP, Zhou Z, Galano A, Vriend J, Tan DX \& Reiter RJ 2015 Melatonin: an ancient molecule that makes oxygen metabolically tolerable. Journal of Pineal Research $\mathbf{5 9}$ 403-419. (https://doi.org/10.1111/jpi.12267)

Mehrpour M, Esclatine A, Beau I \& Codogno P 2010 Overview of macroautophagy regulation in mammalian cells. Cell Research 20 748-762. (https://doi.org/10.1038/cr.2010.82)

Moriguchi T, Toyoshima F, Masuyama N, Hanafusa H, Gotoh Y \& Nishida E 1997 A novel SAPK/JNK kinase, MKK7, stimulated by TNFalpha and cellular stresses. EMBO Journal 16 7045-7053. (https://doi.org/10.1093/ emboj/16.23.7045)

Murdoch WJ 1998 Inhibition by oestradiol of oxidative stress-induced apoptosis in pig ovarian tissues. Journal of Reproduction and Fertility 114 127-130. (https://doi.org/10.1530/jrf.0.1140127)

Nishida K, Kyoi S, Yamaguchi O, Sadoshima J \& Otsu K 2009 The role of autophagy in the heart. Cell Death and Differentiation 16 31-38. (https:// doi.org/10.1038/cdd.2008.163)

Ozaki M, Deshpande SS, Angkeow P, Suzuki S \& Irani K 2000 Rac1 regulates stress-induced, redox-dependent heat shock factor activation. Journal of Biological Chemistry 275 35377-35383. (https://doi. org/10.1074/jbc.M005287200)

Park KJ, Lee SH, Lee CH, Jang JY, Chung J, Kwon MH \& Kim YS 2009 Upregulation of Beclin-1 expression and phosphorylation of Bcl2 and p53 are involved in the JNK-mediated autophagic cell death. Biochemical and Biophysical Research Communications 382 726-729. (https://doi.org/10.1016/j.bbrc.2009.03.095)

Pattingre S, Tassa A, Qu X, Garuti R, Liang XH, Mizushima N, Packer M, Schneider MD \& Levine B 2005 BCl-2 antiapoptotic proteins inhibit Beclin 1-dependent autophagy. Cell 122 927-939. (https://doi. org/10.1016/j.cell.2005.07.002)

Pattingre S, Bauvy C, Carpentier S, Levade T, Levine B \& Codogno P 2009 Role of JNK1-dependent Bcl-2 phosphorylation in ceramide-induced macroautophagy. Journal of Biological Chemistry 284 2719-2728. (https://doi.org/10.1074/jbc.M805920200)

Reiter RJ, Mayo JC, Tan DX, Sainz RM, Alatorre-Jimenez M \& Qin L 2016 Melatonin as an antioxidant: under promises but over delivers. Journal of Pineal Research 61 253-278. (https://doi.org/10.1111/jpi.12360)

Rodriguez-Blanco J, Martin V, Garcia-Santos G, Herrera F, CasadoZapico S, Antolin I \& Rodriguez C 2012 Cooperative action of JNK and AKT/mTOR in 1-methyl-4-phenylpyridinium-induced autophagy of neuronal PC12 cells. Journal of Neuroscience Research 90 1850-1860. (https://doi.org/10.1002/jnr.23066)

Serke H, Vilser C, Nowicki M, Hmeidan FA, Blumenauer V, Hummitzsch K, Losche A \& Spanel-Borowski K 2009 Granulosa cell subtypes respond by autophagy or cell death to oxLDL-dependent activation of the oxidized lipoprotein receptor 1 and toll-like 4 receptor. Autophagy 5 991-1003. (https://doi.org/10.4161/auto.5.7.9507)

Shen M, Lin F, Zhang J, Tang Y, Chen WK \& Liu H 2012 Involvement of the up-regulated FoxO1 expression in follicular granulosa cell apoptosis induced by oxidative stress. Journal of Biological Chemistry 287 25727-25740. (https://doi.org/10.1074/jbc.M112.349902)

Shen M, Liu Z, Li B, Teng Y, Zhang J, Tang Y, Sun SC \& Liu H 2014 Involvement of FoxO1 in the effects of follicle-stimulating hormone on inhibition of apoptosis in mouse granulosa cells. Cell Death and Disease 5 e1475. (https://doi.org/10.1038/cddis.2014.400)
Shen M, Jiang Y, Guan Z, Cao Y, Sun SC \& Liu H 2016 FSH protects mouse granulosa cells from oxidative damage by repressing mitophagy. Scientific Reports 6 38090. (https://doi.org/10.1038/srep38090)

Shen M, Jiang Y, Guan Z, Cao Y, Li L, Liu H \& Sun SC 2017 Protective mechanism of FSH against oxidative damage in mouse ovarian granulosa cells by repressing autophagy. Autophagy 13 1364-1385. (https://doi.or g/10.1080/15548627.2017.1327941)

Soares JM Jr, Masana MI, Ersahin C \& Dubocovich ML 2003a Functional melatonin receptors in rat ovaries at various stages of the estrous cycle. Journal of Pharmacology and Experimental Therapeutics 306 694-702. (https://doi.org/10.1124/jpet.103.049916)

Soares JM Jr, Simoes MJ, Oshima CT, Mora OA, De Lima GR \& Baracat EC $2003 b$ Pinealectomy changes rat ovarian interstitial cell morphology and decreases progesterone receptor expression. Gynecological Endocrinology 17 115-123. (https://doi.org/10.1080/gye.17.2.115.123)

Sui X, Chen R, Wang Z, Huang Z, Kong N, Zhang M, Han W, Lou F, Yang J, Zhang Q et al. 2013 Autophagy and chemotherapy resistance: a promising therapeutic target for cancer treatment. Cell Death and Disease 4 e838. (https://doi.org/10.1038/cddis.2013.350)

Tamura H, Nakamura Y, Korkmaz A, Manchester LC, Tan DX, Sugino N \& Reiter RJ 2009 Melatonin and the ovary: physiological and pathophysiological implications. Fertility and Sterility 92 328-343. (https://doi.org/10.1016/j.fertnstert.2008.05.016)

Tamura H, Takasaki A, Taketani T, Tanabe M, Kizuka F, Lee L, Tamura I, Maekawa R, Asada H, Yamagata Y et al. 2013 Melatonin as a free radical scavenger in the ovarian follicle. Endocrine Journal 60 1-13. (https://doi. org/10.1507/endocrj.EJ12-0263)

Tilly JL \& Tilly KI 1995 Inhibitors of oxidative stress mimic the ability of follicle-stimulating hormone to suppress apoptosis in cultured rat ovarian follicles. Endocrinology 136 242-252. (https://doi.org/10.1210/ endo.136.1.7828537)

Tsujimoto Y \& Shimizu S 2005 Another way to die: autophagic programmed cell death. Cell Death and Differentiation 12 (Supplement 2) 1528-1534. (https://doi.org/10.1038/sj.cdd.4401777)

Vallyathan V 2002 Third International Conference on Oxygen/Nitrogen Radicals: cell injury and disease. Morgantown, West Virginia, June 1-5, 2002. Antioxidants and Redox Signaling 4 957-960. (https://doi. org/10.1089/152308602762197498)

Vega-Naredo I, Caballero B, Sierra V, Garcia-Macia M, de GonzaloCalvo D, Oliveira PJ, Rodriguez-Colunga MJ \& Coto-Montes A 2012 Melatonin modulates autophagy through a redox-mediated action in female Syrian hamster Harderian gland controlling cell types and gland activity. Journal of Pineal Research 52 80-92. (https://doi.org/10.1111/ j.1600-079X.2011.00922.x)

Wei Y, Sinha S \& Levine B 2008 Dual role of JNK1-mediated phosphorylation of BCl-2 in autophagy and apoptosis regulation. Autophagy 4 949-951. (https://doi.org/10.4161/auto.6788)

Weng Q, Liu Z, Li B, Liu K, Wu W \& Liu H 2016 Oxidative stress induces mouse follicular granulosa cells apoptosis via JNK/FoxO1 pathway. PLOS ONE 11 e0167869. (https://doi.org/10.1371/journal. pone.0167869)

Weston CR \& Davis RJ 2007 The JNK signal transduction pathway. Current Opinion in Cell Biology 19 142-149. (https://doi.org/10.1016/j. ceb.2007.02.001)

Zhou YY, Li Y, Jiang WQ \& Zhou LF 2015 MAPK/JNK signalling: a potential autophagy regulation pathway. Bioscience Reports 35 e00199. (https:// doi.org/10.1042/BSR20150167)

Received 21 October 2017

First decision 6 November 2017

Revised manuscript received 20 January 2018

Accepted 23 January 2018 